\title{
Gegen den Willen des Patienten Zwangsbehandlung psychisch Kranker
}

\author{
Die Zwangsbehandlung einwilligungsunfähiger psychiatrischer Patien- \\ ten ist in Deutschland derzeit verboten - auch wenn sie medizinisch \\ nötig wäre. Nun hat der Bundestag einen Gesetzentwurf gebilligt, der \\ das Problem beheben soll.
}

Derzeitige Lage Wehrt sich ein einwilligungsunfähiger psychisch Kranker gegen eine ärztliche Maßnahme, die Arzt und Betreuer zu seinem Wohl anordnen, darf man den Patienten nicht behandeln. Das ist die Konsequenz aus aktuellen Urteilen des Bundesverfassungsgerichts und

\section{Interview}

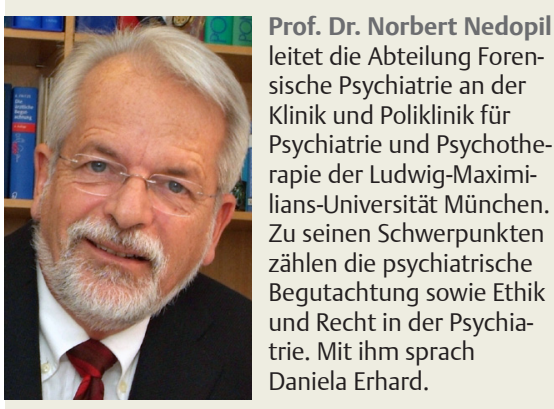

\section{Unterm Strich eine gute Lösung}

? Herr Professor Nedopil, am 17. Januar 2013 hat der Bundestag beschlossen, die Zwangsbehandlung psychisch Kranker neu zu regeln. Was wird sich damit ändern?

Wir befanden uns in einer sehr unsicheren Lage, nachdem das Bundesverfassungsgericht und der Bundesgerichtshof die Gesetze zur Unterbringung und Zwangsbehandlung als nicht verfassungskonform beurteilt haben. Für uns war das Behandeln seitdem eine Gratwanderung zwischen Körperverletzung und unterlassener Hilfeleistung. Mit der Änderung wird die Zwangsbehandlung nun in engen Grenzen wieder erlaubt.

\section{? Löst das die Probleme der behan- delnden Ärzte denn wirklich?}

Zum Einen: Ja, denn es ist geregelt, was erlaubt ist und was nicht - das schafft die Sicherheit, die uns zuletzt gefehlt hat. Allerdings ist das Vorgehen, verglichen des Bundesgerichtshofs [1-3]. Sie erklärten das geltende Recht als verfassungswidrig.
Medizinisch-juristische Gratwanderung Nach dem Betreuungsrecht (§1906 Bür- gerliches Gesetzbuch; BGB) sind daher

mit dem Zustand von 2011, für Ärzte deutlich schwieriger geworden.

\section{? Für die Ärzte ist es schwerer ge- worden? Inwiefern?}

Das Verfahren ist komplizierter. Früher genügte es, wenn der Betreuer eines psychisch kranken und einwilligungsunfähigen Patienten (mit Zustimmung des Betreuungsgerichts) entschied, ob eine medizinisch indizierte Behandlung erfolgen soll oder nicht. Jetzt müssen eine Reihe von Voraussetzungen vorliegen und die Entscheidungen strenger kontrolliert werden.

\section{? Gewisse Voraussetzungen gab es in der früheren Fassung des}

$\S 1906$ BGB aber auch schon.

Die sind aber erweitert worden. Folgende 6 Bedingungen müssen jetzt erfüllt sein:

- Der Patient ist stationär untergebracht. Das Gesetz gilt nämlich nicht für ambulante Patienten.

- Der Patient ist aufgrund seiner Krankheit weder einsichts- noch einwilligungsfähig.

- Die Behandlung ist zum Wohle des Patienten erforderlich, um einen erheblichen gesundheitlichen Schaden von ihm abzuwenden.

- Letzteres kann nicht auf andere Art und Weise geschehen.

- Der Nutzen der Behandlung überwiegt die möglichen Nachteile.

- Sie müssen vorher erfolglos versucht haben, den Patienten zu der Therapie zu motivieren - und haben das dokumentiert.
- v.a. eine Therapie mit Psychopharmaka oder eine Elektrokrampftherapie aktuell nicht möglich, selbst wenn der Patient sich selbstgefährdend verhält oder suizidgefährdet ist.

Für den Arzt ein Dilemma: Verabreicht er ein dringend nötiges Medikament, begeht er eine Körperverletzung - tut er es nicht, macht er sich eigentlich der unterlassenen Hilfeleistung schuldig.

- Freiheitsentzug und Fixierung gelten nicht als ärztliche Maßnahme (Behandlung) und sind daher weiterhin erlaubt.

\section{? Was gilt denn als „erheblicher gesundheitlicher Schaden"? Das} klingt sehr schwammig.

Bestimmt müssen Richter in Zukunft noch klären, was im Einzelfall unter diesen Begriff fällt. Allerdings definiert man hier absichtlich eher offen. Ansonsten fielen ja alle Schäden, die nicht explizit aufgelistet sind, heraus.

\section{? Können Sie ein Beispiel nennen, wann ein solcher Schaden droht?} Bei einem Schizophrenen, der in seinem Wahn akut suizid- oder selbstverletzungsgefährdet ist, kann man wohl davon ausgehen. Schwieriger wird es, wenn es um die Behandlung einer chronischen Erkrankung an sich geht. Bleiben wir bei der Schizophrenie: Die lässt sich medikamentös nicht ursächlich beheben. Jetzt benehmen sich die betroffenen Patienten aber oft unangemessen, rennen beispielsweise in Unterwäsche auf die Straße oder verzocken ihr Geld. Das könnten Sie mit Medikamenten in den Griff kriegen. Doch beginnt da schon gesundheitlicher Schaden?

\section{? Und wie ist das mit der „Überzeugungsarbeit“?}

Ähnlich wie beim Gesundheitsschaden ist es auch hier wenig sinnvoll, allzu enge Vorgaben zu machen. Wie intensiv und lange Sie versuchen müssen, den Patienten zum Mitmachen zu motivieren, hängt vom Einzelfall ab. Bei einem akut lebensgefährlichen Notfall, wie dem katatonen Stupor, wird das meist kürzer sein als bei einer Nahrungsverweigerung. 


\section{Geplante Änderung des Betreuungsrechts}

BGB und FamFG werden nachgebessert Um diese Unsicherheit bei der medizinischen Versorgung betreuter Patienten zu beseitigen, hat der Bundestag im Januar 2013 für einen neuen Gesetzentwurf gestimmt (Drucksache 17/11513). Er legt fest, unter welchen besonderen Voraussetzungen eine Zwangsbehandlung erfolgen darf. Dafür sollen die relevanten Passagen im BGB sowie im FamFG (Gesetz über das Verfahren in Familiensachen und in den Angelegenheiten der freiwilligen Gerichtsbarkeit) ergänzt werden. Das betrifft hauptsächlich

- die Genehmigung der Unterbringung von Patienten (§ 1906 BGB) sowie

? Angenommen, bei meinem Patienten liegen alle Voraussetzungen vor und der Betreuer willigt ein. Darf ich jetzt behandeln?

Noch nicht. Bevor Sie eine Maßnahme gegen den Willen des Patienten starten dürfen, benötigen Sie die Zustimmung des Betreuungsgerichts. Das Gericht wiederum muss - und das ist neu - für seine Entscheidung das Gutachten eines weiteren, nicht an der Behandlung beteiligten Psychiaters heranziehen. Außerdem darf der Betreuer nicht mehr allein entscheiden, sondern ein sogenannter Verfahrensbetreuer muss die Behandlung ebenfalls befürworten. Die Therapieentscheidung von Arzt und Betreuer wird also erst noch unabhängig kontrolliert.

\section{? Das ist patientenfreundlicher als früher.}

Mit der strikten Regelung will man verhindern, dass Ärzte vorschnell handeln, nach dem Motto: „Den kennen wir schon, das machen wir bei ihm immer so." Das ist gut. Wenn ich Patient wäre, würde ich auch gerne über die Therapie mitentscheiden dürfen. Aber gerade den Kollegen im Nachtdienst erschweren die Vorgaben natürlich die Arbeit: Diese rechtlichen Aspekte kann man im akuten Notfall gar nicht alle berücksichtigen.

\section{? Wo ist dann der Vorteil für die Ärzte?}

Aus meiner Sicht sehr positiv ist die Tatsache, dass endlich die Vollmachten berücksichtigt wurden. Die sind nun ins Gesetz geschrieben worden und bieten eine gro-
- die Genehmigung von freiheitsentziehenden Maßnahmen ( $\S 312$ FamFG) und

- die Kontrolle der Therapieentscheidung von Arzt und Betreuer durch Verfahrenspfleger und Gutachter ( $\$ 1906$ BGB sowie $\S \S 312,321$ und 331 FamFG).

Mehr Sicherheit für Ärzte Zwar zielen diese Änderungen laut Gesetzentwurf v.a. darauf $a b$, die Patientenautonomie und die Selbstbestimmung der Betreuer zu stärken. Aber auch für Ärzte sind die Vorgaben nun klarer geregelt.

- Die wichtigsten Punkte erläutert Professor Norbert Nedopil im Interview auf dieser Doppelseite.
Ab wann gilt die neue Regelung? Das Gesetz muss allerdings noch den Bundesrat passieren, vom Bundespräsidenten unterzeichnet und im Bundesgesetzblatt verkündet werden, bevor es wirksam wird. Bis dahin gilt die jetzige Regelung.

Cave Aktuell gilt noch der alte $\S 1906$ BGB! Behandlungen gegen den „natürlichen Willen“ einwilligungsunfähiger Patienten bleiben also vorerst noch verboten.

Daniela Erhard

\section{Literatur}

1 Bundesverfassungsgericht. Beschluss vom 21.04.2011, Az. 2 BvR 633/11

2 Bundesverfassungsgericht. Beschluss vom 12.10.2011, Az. 2 BvR 633/11

3 Bundesgerichtshof. Beschluss vom 20.06.2012, Az. XII ZB 99/12 ße Chance für Arzt und Patient. Optimal wäre eine Vorsorgevollmacht. Man kann darin erstens eine Person seines Vertrauens benennen, die im Fall der Einwilligungsunfähigkeit entscheiden soll, und zweitens erklären, dass man auch eine Zwangsbehandlung in bestimmten Situationen ausdrücklich wünscht. Letztere sollte man konkret ausformulieren. Das würde auch dem Arzt mehr Handlungssicherheit geben.

\section{? Konnte man das denn vorher nicht auch schon so lösen?}

Nein. Die Patienten konnten zwar bisher auch eine Patientenverfügung oder Vollmacht verfassen. Allerdings hatte der sogenannte natürliche Wille bei einer $\mathrm{Ab}$ lehnung der Behandlung immer Vorrang. Das heißt, die Verfügung verlor ihre Gültigkeit, sobald sich der Patient - auch bei einem akuten Wahn - andersgehend äußerte.

\section{? Sprechen Sie mit Ihren Patienten über die Möglichkeit, eine Be-} treuungsverfügung aufzusetzen?

Das machen wir. Und mit dem neuen Gesetz werden Patienten und Ärzte zumindest indirekt darauf hingewiesen, dass man entsprechende Vollmachten vereinbaren sollte. Derzeit spielen diese allerdings noch keine große Rolle in unserem praktischen Alltag.

Zusammenfassend: Wie sollte ein Arzt bei einem einwilligungsunfähigen psychiatrischen Patienten vorgehen?
- Sofern er noch nicht auf Ihrer Station untergebracht ist, müssen Sie den Patienten aufnehmen - aber nur, wenn es begründet ist.

- Dann wägen Sie ab: Ist die Therapie alternativlos und besser als die Nichtbehandlung? Wenn ja, schlagen Sie dem Patienten die Behandlung vor und klären ihn (und den Betreuer) darüber auf.

- Lehnt der Patient die Therapie ab, müssen Sie Überzeugungsarbeit leisten und den Vorgang dokumentieren.

- Weigert sich der Patient weiterhin, folgt der Antrag beim Betreuungsgericht auf Behandlung gegen den Willen des Patienten.

- Das Gericht bestellt dann Gutachter und Verfahrenspfleger und entscheidet nach etwa 7-10 Tagen, ob und in welcher Form Sie behandeln dürfen. An diese Entscheidung müssen Sie sich halten.

\section{? Muss man darüber hinaus noch etwas beachten?}

Wichtig ist, dass die Therapie nicht beliebig lang dauern darf, sondern nur solange sie erforderlich ist. Fallen die Voraussetzungen weg, muss auch die Zwangsmaßnahme enden. Ansonsten gilt: Man darf 2 Wochen behandeln und kann ggf. nochmals um weitere 2 Wochen verlängern. Nach 6-12 Wochen ist aber in der Regel Schluss. Noch länger wäre auch medizinisch meist nicht zu rechtfertigen.

Beitrag online zu finden unter http://dx.doi. org/10.1055/s-0033-1334117 